\title{
Welcome to a new journal!
}

There is a limit to the transferability of scientific learning between two countries

EDITORIALHimalayan Journal of Sciences 1(1): 1, 2003Download in PDF format

\section{Dayananda Bajracharya}

The good news is that despite many limitations from national to individual level, there is a rise in research activities in Nepal. But the bad news is that the lack of standard and regular scientific journals within the country always discourages researchers when they prepare to publish their findings. This journal should be effective towards meeting the dire need of the researchers.

There is an unmistakable correlation between the research base and productivity growth of a country. This reality has been fully recognized by developed countries and some developing countries such as India, China and Brazil. However, there is a general tendency in most of the developing countries to believe that poor and underdeveloped countries do not have adequate resources to support scientific research. Moreover, many people in these countries tend to believe that enough knowledge is being generated by research in developed countries that can be adopted by developing countries and, therefore, there is no need for developing countries to generate their own independent research base. Such belief ignores the fact that there is a limit to the transferability of scientific learning between one country and another due to the uniqueness of each country. Each country has its own specific problems related to its development that can be solved only by indigenous scientific research. Solutions to a country's problems cannot always be found externally. Each and every country needs to develop the capacity to do its own research. A country without an indigenous scientific and technological capacity has no means of being aware of its own needs, nor of the opportunity existing in the field of science and technology elsewhere.

In Nepal, culture of scientific research has yet to take firm roots. Research is not yet accorded the due priority it deserves. There is still no national policy on research. Investment in research and development is one of the lowest, even by regional standard. Researchers have very few motivations and incentives. They are compelled to work under conditions of extreme limitations. Most laboratories lack even basic minimum requirements. Access to scientific information is hampered due to the limited access to such modern facilities as internet etc. Research is still a ten-to-five business in Nepal. Many highly qualified teachers with great research capability are enticed away from their research activity by monetary attraction to teach in several campuses. There are very few researchers for whom the primary motivation is the urge to discover.

In spite of all these limitations, research activities in Nepal are on the rise compared to few decades ago. This is evidenced by the large number of research papers that are being presented in national and international science conferences held in the country from time to time. One of the major problems faced by the researchers is the lack of standard and regular scientific journals within the country to publish their findings. It is my earnest hope that the present journal will be effective towards meeting this need of the researchers. In the past, many journals have appeared and disappeared in quick succession. I hope that editorial board of Himalayan Journal would strive hard to maintain the quality and the regularity of the journal. I wish them all the success.

Dayananda Bajracharya is a professor of botany in Tribhuvan University and vice Chancellor of Royal Nepal Academy of Science and Technology. E-mail: dbajra@mail.com.np 